University of Nebraska - Lincoln

DigitalCommons@University of Nebraska - Lincoln

2004

Concentrating, purifying and detecting waterborne parasites

Dante S. Zarlenga

James M. Trout

Follow this and additional works at: https://digitalcommons.unl.edu/usdaarsfacpub

This Article is brought to you for free and open access by the U.S. Department of Agriculture: Agricultural Research Service, Lincoln, Nebraska at DigitalCommons@University of Nebraska - Lincoln. It has been accepted for inclusion in Publications from USDA-ARS / UNL Faculty by an authorized administrator of DigitalCommons@University of Nebraska - Lincoln. 


\title{
Review
}

\section{Concentrating, purifying and detecting waterborne parasites}

\author{
Dante S. Zarlenga, ${ }^{\mathrm{a}, *}$, James M. Trout ${ }^{\mathrm{b}}$ \\ ${ }^{a}$ U.S. Department of Agriculture, ARS, Bovine Functional Genomics Laboratory, Building 1180, \\ BARC-East, Beltsville, MD 20705, USA \\ ${ }^{\mathrm{b}}$ U.S. Department of Agriculture, Environmental Microbial Safety Laboratory, Beltsville, MD 20705, USA
}

\begin{abstract}
There has been recent emphasis on developing better methods for detecting diseases of zoonotic and veterinary importance. This has been prompted by an increase in human disease agents detectable in environmental samples, the potential for bioterrorism, and the lowering of international trade barriers and expansion of personal travel, which are bringing previously considered exotic diseases to new geographical localities. To appreciate the complexities of developing detection methods and working with environmental samples, it is appropriate to review technologies currently in use, as well as those in development and presently limited to research laboratories. Discussion of parasite detection would not be possible without including methods for parasite sampling, concentration, and purification because it is often necessary to process large sample volumes prior to analysis, and no reliable methods are available for significantly amplifying parasites in vitro. Reviewing proven methods currently in use will provide a baseline for generating, accepting and implementing the more sensitive and specific methods under development today.

Published by Elsevier B.V.
\end{abstract}

Keywords: Waterborne parasites; Detection; Identification; Cryptosporidium; Giardia

\section{Contents}

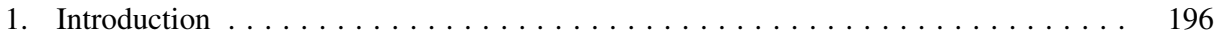

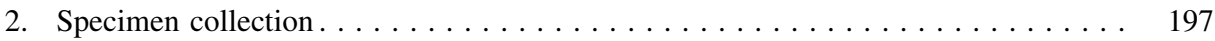

* Corresponding author. Tel.: +1 301504 8754; fax: +1 3015048979.

E-mail address: zarlenga@anri.barc.usda.gov (D.S. Zarlenga). 
3. Concentration methods . . . . . . . . . . . . . . . . . . . . . . . . . . 199

3.1. Sedimentation techniques . . . . . . . . . . . . . . . . . . . . . . . . . 199

3.1.1. Flocculation . . . . . . . . . . . . . . . . . . . . . . 199

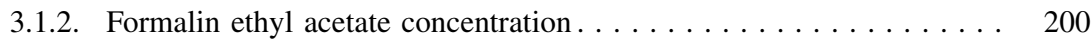

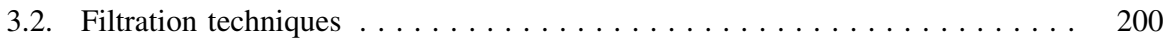

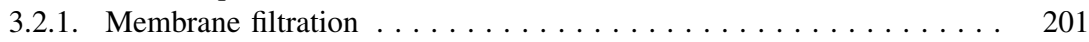

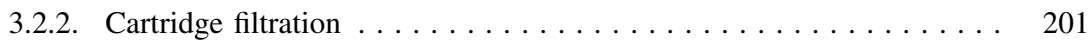

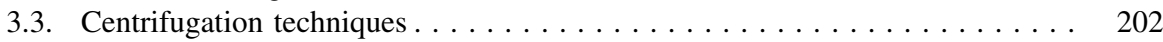

4. Purification methods. . . . . . . . . . . . . . . . . . . . . . . . . . 203

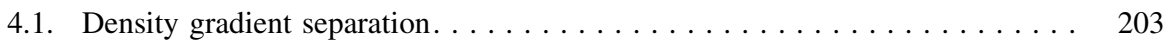

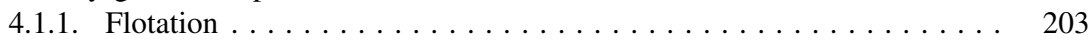

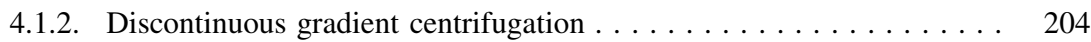

4.2. Immunomagnetic separation . . . . . . . . . . . . . . . . . . . . . 204

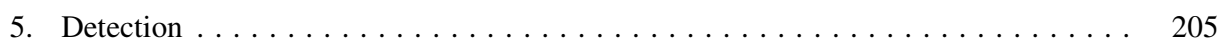

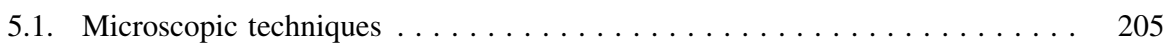

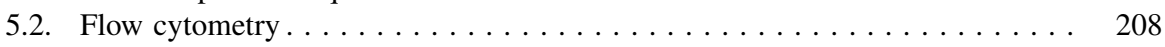

5.3. Molecular techniques........................... 209

6. Methodologies on the horizon $\ldots \ldots \ldots \ldots \ldots \ldots \ldots \ldots \ldots \ldots \ldots \ldots \ldots \ldots$

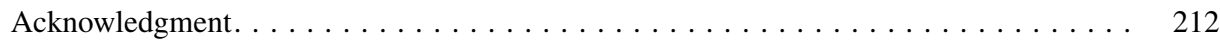

References .............................. 212

\section{Introduction}

In recent years, parasite identification and detection have taken on new roles and responsibilities. This has resulted from an increase in our understanding of the complex structure of parasite genera and the need to further delineate genotypes within species. Coupling this information with the increasing demand for safer living environments, the health risks that pathogenic parasites impart on immunocompromised individuals, and the looming potential for using zoonotic organisms in bioterrorism, accurate characterization is not only required to identify the source(s) of infection and implement proper controls, but is necessary to properly treat the associated human infections. As a result, we have seen a revolution in the numbers and types of methodologies for detecting parasites that complement currently established methods.

In evaluating detection procedures, one must delineate between those commercially proven for analyzing environmental samples, those in various stages of development and those useful in research laboratory settings. It is the third group, which is a broad and evolving class of detection methods that is the focus of this report. In general, identification and detection are direct methods primarily developed to define presence or absence, and to discriminate among parasite groups. Diagnostic techniques, on the other hand, can be 
either direct or indirect in nature and tend to define the disease state of an individual or animal. In some cases identification, detection and diagnosis are mutually exclusive; however, the examples to be presented herewith will concentrate on direct analysis and environmental sampling, and their relationship to sample collection, assay sensitivity, reproducibility, and specificity, rather than to define the health status of the host. Furthermore, the discussion at times will elaborate on those parasites that currently pose the greatest health risks and are widely disseminated in our environment.

Parasite identification methods can be arbitrarily classified into three broad categories: general morphology, chemical and immunofluorescent stains, and biochemical and molecular biological tests. As emerging tests report greater sensitivity and specificity, it is important to note the overall trend toward greater objectivity where data interpretation relies less on the trained eye and more on instrumentation for final assignment. As with all detection methods, sensitivity is a relative issue. A reliable test cannot be developed before the limitations of the problem are defined. This in itself is a major downfall of many approaches to assay development. Since sensitivity levels asymptotically approach a theoretical detection limit i.e.100\%, it is an absolute imperative to determine what level of parasite contamination is acceptable in an environmental sample before attempting to develop a reliable test. Agreeing on the acceptable presence of any etiologic agent is a formidable task. Often times, modifying the method of sample collection to concentrate the target organism can greatly assist in shifting the sensitivity level closer to the theoretical maximum. Methods of sample collection, concentration and purification will be presented as a prelude to discussing various parasite detection methods.

A wide-range of technologies have been used to detect and differentiate parasites; however, as progress is made toward developing the most sensitive assays we have come to rely on DNA analysis. Molecular-based identification methods that focus on the parasite genome have the distinct advantage of not being limited to any one particular developmental stage. Other than for chromatin diminution which occurs very early in the development of some parasitic nematodes (Boveri, 1887), the integrity of parasite DNA is essentially invariant throughout its life-cycle and does not succumb to short term environmental stress factors that can alter the results of protein-based methods. DNA analysis has been both a boon and bust for those working in the field of parasite detection. Clearly, technologies can now differentiate to the level of unique genotypes within single populations of organisms; however, this mastery often outpaces our abilities to ascribe biological events or characters to these differences. Consequently, increased specificity must be accompanied by a utilitarian application. DNA-based advancements made in parasite detection will be examined and newer technologies in the areas of high throughput screening will be discussed.

\section{Specimen collection}

Protozoans are among the most common parasitic pathogens present in environmental samples. This parasite group exhibits multi-stage life cycles, consisting of an environmentally resistant stage (oocyst, cyst, or spore) excreted in feces that is capable of infecting new hosts, and an intermediate or tissue stage that replicates within the host. 
Toxoplasma gondii and related tissue-cyst-forming protozoa such as Sarcocystis spp. are among a small group of parasites transmissible through ingestion of either infected tissues or the oocyst (environmental stage). Of over 350 vertebrate host species, only felids produce $T$. gondii oocysts, whereas with many other parasites, virtually all hosts are capable of producing infective environmental stages. Thus, when deciding on a detection strategy, it is necessary to determine if assays will be targeted at tissue/intermediate stages and/or environmental stages. Additionally, an infected host will usually produce antibodies against a parasitic agent; however, detection of host antibodies or parasite antigens are not necessarily indicative of the presence of the environmental stages and are beyond the scope of this article. Other considerations include the necessity to distinguish between viable and non-viable parasites as well as identifying particular species or genotypes of a parasite. In environmental samples, presence and not viability is more often the critical factor. Species and genotypic differentiation are frequently not part of the more common detection methods; however, with the identification of genotypic variants of Giardia (Thompson et al., 2000) and Cryptosporidium (Xiao et al., 2000), which differ in their proclivity for human hosts, more refined molecular detection methods are now under development.

Approaches to sample collection and processing are often dictated by the choice of the ensuing test. Water or soil might be collected to determine the levels of parasites present in the environment, or in the case of a water treatment facility to ensure that parasite-free drinking water is being provided. Some of the problems associated with determining the limits of parasite detection in potable water are clearly exemplified by the markedly different quantities of water that should be collected before analysis as defined by the US Federal Register Requirements for Public Drinking Water Supplies and those suggested by the American Society for Testing and Materials (ASTM). In addition to quantities, the frequency of analysis also can vary, e.g. most procedures consider batch rather than continuous analysis to be sufficient. These examples illustrate the problem in identifying a level of environmental presence (an acceptable risk level) that would be tolerated by the average immunocompetent individual. Unfortunately, these numbers tend to be arbitrarily determined and for this reason, it has been difficult to mandate analysis of water samples. Requirements to monitor unpasteurized juices and milk, and raw fruits and vegetables for parasites would likely run into similar regulatory problems.

Feces are normally collected to determine the infection status of the host; however, evaluating feces as the source of environmental contamination or estimating the prevalence of a given parasite group within a geographical area are topics more germane to the discussion at hand. Generally, the larger the volume of the sample, the greater the chances for parasite detection following concentration; however, processing large volumes can be labor-intensive, time-consuming, costly, and can result in increased levels of contaminants which may interfere with subsequent detection methods. Whenever possible, fecal samples should be obtained directly from the animal to minimize the risk of cross-contamination, and prevent the introduction of parasites by insects. Numerous insects are known to be infected with microsporidia species that are not infectious to mammals (Becnel and Andreadis, 1999; Sprague and Becnel, 1999), and filth flies have been shown to transport C. parvum oocysts (Graczyk et al., 1999, 2000). Thus, feces collected from the ground should be discarded if there is obvious evidence of insect activity. Ideally fecal samples should be processed within a few hours of collection or stored cold to prevent loss of 
moisture, overgrowth by fungi, and deterioration of antigen or DNA. Many parasites can be killed quickly by freezing or drying, and viability tends to decrease more rapidly at higher temperatures (Anderson, 1986; deRegnier et al., 1989; Fayer et al., 1998; Fayer and Nerad, 1996; Robertson et al., 1992). Long storage times increase the risk of excessive growth of molds, bacteria, and free-living microorganisms, and the effects of large numbers of these other organisms on parasite concentration and detection are not well known.

\section{Concentration methods}

Each sample type presents unique challenges in parasite isolation, concentration, and detection. Depending on the source, water can contain large amounts of silt, humic acid, decaying organic material, and free-living organisms (bacteria, protozoa, algae). Feces contain mucus, lipid, bacteria, fungi, insects, pollen and partially digested foodstuffs. These materials can clog filters, trap parasites during flotation, disrupt gradient formation, and inhibit PCR reactions. On the other hand, a certain level of particulate matter $(\sim 10$ nephelometric turbidity units (NTU)) of the correct size $(5-40 \mu \mathrm{m})$ can actually increase C. parvum oocyst recovery from water by acting as a carrier (Feng et al., 2003).

Soils have the ability to bind oocysts, cysts and spores; however, the efficiency of this binding varies with the geographical locality and soil type. Binding of the parasite to soil particles can significantly decrease recovery yields. C. parvum oocyst recovery from soil was shown to negatively correlate with clay content (Kuczynska and Shelton, 1999) although addition of a mild surfactants i.e. Tween 80, Triton X-100, improved recoveries. For these reasons and the obvious low levels of parasite often found in environmental samples, microscopic analysis is often preceded by a concentration step such as sedimentation, filtration or centrifugation. Sedimentation and filtration have the added benefit of removing large particulate matter that can interfere with subsequent detection.

\subsection{Sedimentation techniques}

Sedimentation relies upon the relative weight of the parasite to partially separate it from more buoyant impurities and particulate matter. In the simplest case, a water sample would be placed into a tube, subjected to centrifugation, and the resulting pellet tested by one of the established detection methods. Unfortunately sample processing is rarely this straightforward and more involved techniques are often required to provide a cleaner more concentrated sample.

\subsubsection{Flocculation}

Flocculent settling, or flocculation, refers to a dilute suspension of particles that coalesce in solution. During this process, the particles increase in mass and settle at a faster rate. This process transforms dissolved or suspended matter into an insoluble solid, facilitating its subsequent removal by centrifugation, sedimentation or filtration. Most often, the $\mathrm{pH}$ is adjusted and a chemical precipitant is added which creates the flocculent. After the precipitate has settled, the supernatant fluid is aspirated, acid or base is then added to dissolve the flocculent, and the sedimented material (containing the parasites) is 
suspended in water. Vesey et al. (1993a) tested a number of potential flocculents including beef extract paste, skim milk powder, alum (aluminum sulfate), ferric sulfate, ferrous sulfate, ferric chloride and calcium carbonate. Recovery efficiencies using flocculents for concentrating Cryptosporidium oocysts, were reported to be 59\% for alum (1000 oocysts/ L) (Vesey et al., 1993a) and 64-75\% for calcium carbonate (75-1000 oocysts/L) (Vesey et al., 1993a; Campbell et al., 1994; Shepherd and Wyn-Jones, 1996). Giardia cysts recoveries were $72-77 \%$ using calcium carbonate (Shepherd and Wyn-Jones, 1996) on river or tap water seeded with 75 oocysts or 85 cysts/L. Recently, Tsushima et al. (2003) successfully used ferric sulfate as the flocculent to detect $C$. parvum in several natural water supplies of Hokkaido, Japan. Because flocculation requires $\mathrm{pH}$ adjustments that are substantially beyond physiological ranges, parasite viability may be compromised during the isolation process (Campbell et al., 1994). In addition, precipitation of particulates other than parasites can present substantial debris in the final preparation and cause poor recoveries. The original procedures recommended allowing solutions to settle undisturbed overnight; however, centrifugation makes testing more rapid and may improve sample recoveries (Shepherd and Wyn-Jones, 1996). It has been suggested that variations in the hydrophobic and electrostatic characteristics of protozoan surfaces can impact the efficiency of recoveries using flocculation and that prior knowledge of cell surface makeup may assist in choosing among the many flocculents available (Drozd and Schwartzbrod, 1996; Hsu et al., 2001b).

\subsubsection{Formalin ethyl acetate concentration}

Formalin ethyl acetate concentration is a technique routinely used for processing fecal samples. The procedure is predicated upon differences in specific gravity between the parasitic forms and the ethyl acetate. Ethyl acetate concentration recovers all protozoa, eggs, and larvae present in a sample; however, the resultant preparation contains substantially more debris than flotation, which is used more for final purification. When used with Triton X-100, the ethyl acetate will also remove fats and oils from the sample, though if this is a big problem the ethyl acetate can be replaced with ether. Polyvinyl alcohol-preserved specimens can also be used. After final centrifugation, formalin fixed parasites will be present in the pellet leaving much of the fecal debris in the aqueous layer. A simple modification of this technique using a merthiolate-iodine-formaldehyde mixture preceded by treatment with $10 \%$ formalin and filtering through a double-layered cotton filter substantially reduced fecal debris in the final sample (Wang, 1998). Formalin ethyl acetate concentration has the advantage of being rapid, inexpensive and amenable to handling large numbers of samples simultaneously; however, formalin fixation will render parasites non-viable, potentially distort cellular exudate, destroy trophozoite stages and often interfere with antibody-based detection methods though sufficient DNA can usually be purified for detection by PCR. Also, parasites may be lost during wash steps and fecal debris is not completely removed.

\subsection{Filtration techniques}

Membrane or cartridge-based filtration techniques are most often used to remove microscopic organisms from water samples, although they can be used also to remove such 
organisms from dilute slurries of fecal or environmental matter. Currently, cartridge filtration is integral to the United States Environmental Protection Agency (USEPA) Methods 1622 and 1623 for detection of Cryptosporidium and Giardia in water (USEPA, 1998, 1999). Depending on the organisms and method of detection, membrane and not cartridge filtration have been shown to result in higher recoveries and detection limits (Hsu et al., 2001a).

\subsubsection{Membrane filtration}

Membrane filtration, which utilizes large diameter (142 or $293 \mathrm{~mm}$ ) flat filters, has been described for the concentration of $C$. parvum oocysts from water samples (Ongerth and Stibbs, 1987), and has been used for Giardia as well. Water is pumped through the membrane and the retained materials are recovered by scraping and centrifugation. Alternatively, newer cross-flow or tangential-flow membrane filtration methods (Brandonisio et al., 2000) which showed promising results for Giardia and Cryptosporidium have been tested as ways to minimize contaminant buildup at the membrane surface and increase flow rates. Although membrane cleaning is periodically required, the self-cleaning nature of cross-flow filtration lengthens membrane life to make it economically attractive. Vortex-flow filtration (Fricker et al., 1997) which suppresses filter clogging by the vortices generated during the filtration process by a rotating membrane has produced relatively consistent oocyst recoveries of $60-90 \%$ in seeded samples, though seeding densities were not disclosed. Vortex-flow filtration can require longer processing times compared to the standard cartridge method and results, though reproducible, are highly dependent upon operator experience. In general, normal membrane filtration can easily filter 10-40 L of low-turbidity water, while filtration of high-turbidity waters is limited to only 1-2 L. A range of recovery efficiencies has been described for flat-bed membranes where Nieminski et al. (1995) reported average recoveries of $9 \%$ for Cryptosporidium and $49 \%$ for Giardia for concentrations at 25 oocysts/L for each parasite group. Recovery efficiencies of different parasites, however, can be influenced by the choice of filter material. Shepherd and Wyn-Jones (1996) reported that when $100 \mathrm{~L}$ samples were seeded with 75 oocysts or 85 cysts/L, cellulose-acetate membranes gave higher recovery rates (30-40\% for Cryptosporidium and 50-67\%, for Giardia) than the polycarbonate membranes (22-36 and 41-49\%, respectively) tested by Ongerth and Stibbs (1987) using seeding densities of 50-500 oocysts/L. Unfortunately, the simple, rapid and inexpensive nature of membrane filtration in conjunction with its ability to process large volumes of water with high yield recoveries is negated by the clogging of membranes that occurs with high-turbidity samples. Thus, subsequent sample purification is often required before detection can be performed.

\subsubsection{Cartridge filtration}

Cartridge filtration was developed as an alternative to filtration through flat membranes. Cartridge filters, containing spun polypropylene, with a nominal pore size of $1 \mathrm{~mm}$ were among the first used to detect Cryptosporidium in water (Musial et al., 1987) where large volumes of water $(100-1000 \mathrm{~L})$ could be filtered at high flow rates $(1-5 \mathrm{~L} / \mathrm{min})$. Retained material was then eluted by cutting open the filter and washing it with a dilute detergent solution followed by centrifugation. First reports showed a recovery of 9-29\% for 
Cryptosporidium oocysts seeded at low densities (100-1000 oocysts/378 L) (Musial et al., 1987); however, both lower efficiencies (<1-30\%) (Clancy et al., 1994; Ongerth and Stibbs, 1987; Shepherd and Wyn-Jones, 1996) and higher efficiencies (75\%) (Clancy et al., 1997) have since been reported. As with most techniques, recovery rates can be affected by a number of factors including water quality, and oocyst age (Hsu, 2003; Hsu et al., 2001a). Nonetheless, in 1999, this technique garnered the interest of the USEPA and in an effort to improve on the information collection requirement (ICR) methods for detecting Cryptosporidium and Giardia, the EPA recommended Method 1623. Method 1623 is still subject to turbidity problems but less so than its predecessors by following cartridge filtration with immunomagnetic separation to provide a cleaner sample for final examination. This permits reducing the sample size from 100 to $10 \mathrm{~L}$, but in so doing reduces the detection limit. Simmons et al. (2001) and Kuhn and Oshima (2002) showed that a disposable hollow-fiber, ultra-filtration system exhibited equal or better efficiency than the capsule filter system used in Method 1622; however, the time to process comparable water samples was excessive, albeit cost-effective. Even laboratory-scale sand columns have been tried with reasonable success using hydraulic loadings at $5 \mathrm{~mL} / \mathrm{min}$ (Whitmore and Carrington, 1993); however, unsatisfactory recoveries (down to 2.3\%) resulted when the flows were increased to more practical rates obtainable with the cartridge-based methods. It is interesting to note that work by Feng et al. (2003) suggests that the turbidity of the water may actually increase parasite recoveries, though solutions too high in particulate matter can clog filters, reduce flow rates and require additional work to dissect and wash the filters to release the parasites.

\subsection{Centrifugation techniques}

The link between centrifugation techniques and processing environmental samples is multi-faceted. Centrifugation is used both for sample concentration and purification. When used for parasite concentration, conditions such as speed, volume and time must be empirically determined and these variables will change with the make-up of each environmental sample. This simplistic approach to parasite concentration is easily implemented in any lab with access to a preparative centrifuge, provided small sample volumes will be analyzed; however, classical centrifugation using tubes or bottles is not well suited to processing large volumes of liquid which is becoming the focus of modern technologies. For this reason, continuous flow centrifugation (CFC) was developed where particulate matter is collected from large volumes of liquid via uninterrupted centrifugation until the volume of pelleted material exceeds the rotor capacity. Continuous flow centrifugation can also be used for density gradient separation to process fecal slurries allowing the more buoyant parasites to migrate to the top of the gradient. In these cases, the resultant supernatant containing the parasites is diluted to reduce the density then subjected to a second pass through the centrifuge to concentrate the parasites at the bottom of the rotor. Such a step may preclude the need for subsequent purification. Whitmore and Carrington (1993) first evaluated CFC and showed relatively poor recoveries (11-31\%). More recently, Higgins et al. (2003) successfully used CFC followed by immunomagnetic separation to concentrate and purify $C$. parvum oocysts from spiked water samples, claiming this technology as a rapid and economical alternative to capsule-filtration based 
methods. Zuckerman et al. (1999) earlier developed a portable CF centrifuge potentially suitable for field analyses that was compact, mobile, flexible, and generated reproducibly higher recovery rates. The most common CFC techniques mentioned above involve bowl centrifuges. Recently, a CFC procedure was developed by Borchardt and Spencer (2002) proclaiming higher and more reproducible recoveries using a channel centrifuge. The biggest advantage of this technique was the processing of large volumes of aqueous suspensions unencumbered by high concentrations of sample debris; however, the method required substantially lower flow rates to achieve efficient separation of particulates from the solution and therefore was deemed too time-consuming for conventional application.

As mentioned earlier, centrifugation methods can be utilized at different points in sample processing. Techniques such as flotation and density gradient centrifugation, though adaptable to concentrating parasites, are more often used to purify previously concentrated organisms. As such, these techniques are appropriately discussed below in light of their more universal application to purify parasites.

\section{Purification methods}

Parasite concentration must usually be followed by a purification step prior to using sensitive detection methods. The most common techniques involve density gradient centrifugation, which takes advantage of differential densities between the parasites and the debris for purification. Other more sophisticated procedures use parasite-specific antibodies coupled to magnetic particles. Magnetic separation techniques have become commonplace with the acceptance of USEPA Methods 1622 and 1623.

\subsection{Density gradient separation}

Density gradient separation uses solutions of high specific gravity to float parasites and pellet heavier debris. In some circumstances, densities may be altered to float lighter debris and pellet the parasites. While generally not suitable to processing extremely large sample volumes other than for $\mathrm{CFC}$, these techniques are capable of yielding parasite preparations that are relatively free of contaminating material.

\subsubsection{Flotation}

Flotation is the simplest form of gradient separation where an aqueous sample is mixed with or layered over a dense solution at a predetermined specific gravity and subjected to centrifugation. A variety of solutions have been used to effect separation including: sucrose, sodium chloride, cesium chloride potassium bromide, zinc sulfate and percoll. When using zinc sulfate, it was observed that centrifugation was not always necessary (Zajac et al., 2002) in that the lower density of the parasites relative to their surrounding medium naturally caused them to float when permitted ample time (5-10 min). Anecdotal evidence suggests that similar results can be achieved with sucrose as well. This, however, needs to be empirically determined with each parasite group and may not be suitable for protozoans. Also, Malicki et al. (2001) used known numbers of colored helminth eggs spiked into samples prior to concentration and purification to monitor flotation recovery 
efficiencies and delineate these from naturally occurring eggs. This approach has yet to be tested for other parasite groups. Mes et al. (2001) demonstrated that in some circumstances improvements on the Wisconsin flotation method can be achieved using a 'salt-sugar' approach that begins with a salt gradient followed by a sugar flotation to improve recoveries. Unfortunately, flotation may selectively purify viable, intact parasites and skew data that relies on recovery percentages rather than viability.

\subsubsection{Discontinuous gradient centrifugation}

Discontinuous gradient centrifugation is similar to flotation. In this instance, solutions of decreasing specific gravities are layered by pipetting into a centrifuge tube rather than using one solution of high density as in flotation. The aqueous suspension containing parasites, is then layered on top of the gradient and centrifuged. If centrifuged at the appropriate $g$-force for a specified period of time, the parasites will form a band at a density that separates them from both heavier and lighter contaminants. Prior knowledge of the approximate density of the parasites is beneficial to reduce trial and error. C. parvum oocysts can usually be separated in a discontinuous $\mathrm{CsCl}$ gradient ranging from 1.05 (top) to $1.4 \mathrm{~g} / \mathrm{mL}$ (bottom). After centrifugation at $10,000 \times g$ for $15 \mathrm{~min}$, a relatively pure band of oocysts can be found near the $1.1 / 1.05 \mathrm{~g} / \mathrm{mL}$ interface. Cesium chloride is not the only reagent useful for discontinuous gradient centrifugation. Entrala et al. (2000) were successful in using potassium bromide, a low cost reagent, to purify large amounts of C. parvum oocysts using a standard table-top centrifuge. Clearly, discontinuous gradient centrifugation generates relatively pure parasite preparations when performed carefully; however, small sample volumes must be used and highly concentrated samples will ultimately disrupt gradient formation causing poor recoveries and excessive contamination in the desired product.

Regardless of the reagent, parasite viability has a direct effect on its buoyant density. Bautista et al. (1999) found that formaldehyde-fixed parasites subjected to sucrose gradient centrifugation were present only in the pellet, whereas fresh parasites appeared in both the pellet and in one or more bands where $92-100 \%$ viability was observed depending on the location of the band within the gradient. Bukhari and Smith (1995) showed that purification using water-ether rather than sucrose or zinc sulfate flotation was preferable in epidemiological studies when both Cryptosporidium oocyst enumeration and viability were required because of loss of viability when using flotation techniques.

\subsection{Immunomagnetic separation}

Immunomagnetic separation (IMS) uses antibodies against parasite surface antigens bound to magnetic particles to capture and remove the parasite from the sample using a magnet. Though simple in principle, there are several potential sources of failure, including the specificity and affinity of the antibodies for the parasite and the quality of the beads. When antibody coated beads are mixed with the sample, the bonds between the immunoglobulin and the oocyst are subjected to shear forces during the purification procedure. Thus, the higher the affinity of the antibody for the parasite, the more likely the cyst or oocyst will remain attached to the bead during purification. This can be problematic in that high-affinity binding can sometimes affect release of the parasite from the bead and 
reduce recoveries (Rochelle et al., 1999). This is of particular importance when analyzing environmental samples low in parasite numbers. The way in which the antibody is attached to the bead may also have an effect on recovery efficiencies. Targeted covalent linkages or biotin-avidin interactions provide a strong bond that leaves the antigenic epitope unencumbered to interact with the parasite surface.

The efficiency of IMS is inversely related to the NTU of the sample. Bukhari et al. (1998) found that recoveries of Cryptosporidium ranged as low as $0.2 \%$ and as high as $83 \%$ at seeding densities of 50-87 oocysts/L depending on the purity of the sample and the source of the IMS kit. Others as well have demonstrated substantial disparity in parasite recoveries among commercially available kits (Campbell et al., 1997; Fricker et al., 1997; Rochelle et al., 1999) reaching in excess of $90 \%$ when clean suspensions of C. parvum oocysts were used (Campbell and Smith, 1997; Campbell et al., 1997). The size of the bead and the method of agitation, which is dictated by the shape of the magnet, can impact the efficiency of parasite binding to magnetic beads. Agitation requiring horizontal rocking rather than rotary action gave the highest recoveries whereas circular motions tended to concentrate the heavier magnetic beads in the center of the dish thereby reducing surface contact with the parasite (Rochelle et al., 1999). The use of IMS assays are currently increasing for Giardia (Ferrari and Veal, 2003; Robertson and Gjerde, 2000; Yakub and Stadterman-Knauer, 2000), Cyclospora (Robertson et al., 2000) and microsporidia (Dowd et al., 1998; Sorel et al., 2003). A benefit of this technology is that it permits nondiscriminatory purification of protozoan species by attaching to the magnetic beads antibodies to conserved surface epitopes, then relying on a subsequent PCR assay for species-specific detection. Regardless of the problems associated with turbidity, IMS is fast becoming the preferred method for final purification prior to parasite detection.

\section{Detection}

Microscopy plays a prominent role in the identification of microorganisms, and in all likelihood, will continue to do so in the foreseeable future. In some developing countries, microscopy may be the only tool available for identification. With the advent of PCR and its trailing technologies, new methods for detecting microorganisms are continually evolving. Nonetheless, until commercial acceptance of PCR for detection of parasites becomes more commonplace, microscopic observation of samples will likely remain the method of choice. Other techniques such as ELISA and flow cytometry, which have already undergone industry scrutiny and standardization, have also emerged as valuable detection tools. Thus, an evaluation of detection methods should include both the microscopic methods currently in use, as well as molecular methods in development.

\subsection{Microscopic techniques}

The virtues of simple transmitted light microscopy should not be overlooked in the current era of technological advances. In developing countries where resources are limited, microscopic examination of wet preparations, or of dried, fixed, chemically stained specimens, is the most viable option. Light microscopes can be relatively inexpensive, and 
the basic supplies such as slides and chemical stains are often available at a fraction of the cost of molecular or immunological reagents. Additionally, the preparation of specimens is less complicated and often less time-consuming than those required to analyze samples using more technologically based methods. Even in more developed countries, many individuals prefer making final determinations regarding the presence or absence of parasites based upon their own ability to observe the organism through the microscope.

Perhaps the most critical factor in the use of microscopy as a detection technique is the skill and experience of the microscopist well trained in identifying the environmental stages of parasites. This has been considered both a positive and negative for those performing the analyses in that some contend the subjective eye is still more reliable than PCR assays which can also generate false-positive and/or false-negative results. Microscopic observations can be made using brightfield (with or without special stains), phase contrast, differential interference contrast (DIC, a.k.a. Nomarski), or fluorescence microscopy (for autofluorescing as well as stained specimens).

Visible light techniques (brightfield, phase contrast, DIC) are usually the simplest to use and these are relatively effective in the analysis of parasite eggs; however, brightfield observation of unstained samples for oocysts is often tenuous and is highly dependent upon the purity and concentration of the sample. The small size and lack of refractility makes it very difficult to detect parasites in preparations containing debris and other organisms of similar size, even to the experienced observer. Numerous chemical stains such as hematoxylin, acid fast, Giemsa, Toluidine blue, periodic acid Schiff, various gram stains and others, have been used to aid in parasite detection and recognition; however, these do not always uniformly stain the parasites. In addition, staining quality is dependent upon reagent preparation, staining technique, variability in dye uptake and the ability of other non-parasitic microorganisms and debris to bind stain. Phase contrast and DIC microscopy provide more contrast between specimens and the background. This can often reveal internal morphology and therefore provide better visualization and identification of parasites relative to brightfield when examining unstained samples; however, both of these techniques are best suited for wet preparations, which limits advance preparation of slides prior to inspection. Recently, some researchers have taken advantage of a natural autofluorescence emitted by both nematode and protozoan parasites to enhance detection (Bialek et al., 2002; Daugschies et al., 2001). This approach bridges brightfield techniques and the fluorescent methods described below with a sensitivity level that also resides between both approaches. Autofluorescence present in many algae and other environmental contaminants can prove to be problematic when using fluorescent chemical stains.

Direct staining of parasites with fluorochromes or indirectly but specifically labeling them with antibodies conjugated to fluorochromes (immunofluorescence assays (IFA)) can significantly improve detection and differentiation from unstained background debris when viewed under ultraviolet illumination with filters appropriate to the chosen fluorochrome. In some cases counterstains are used to dye the background debris a contrasting color to enhance detection. Calcofluor White is one such compound that binds to chitin-containing components and is useful in screening for microsporidia spores, although it can only provide a presumptive positive result because it stains yeasts and some bacteria (Fayer et al., 2003). Consequently, the industry has substantially embraced immunofluorescent 
stains such as fluorescein isothiocyanate (FITC) conjugated to primary (direct) or secondary (indirect) antibodies.

Fluorescent staining techniques using labeled antibodies confer significant advantages for detection of parasites. Because the antibodies are specific to a parasite species, fluorescence from debris and other organisms is substantially reduced which in turn requires considerably less training for definitive identification of organisms. Both polyclonal and monoclonal antibodies (MAbs) have been raised against purified parasites and used in immunofluorescent staining. MAbs are generally preferable because of reduced cross-reactivity with non-target organisms and the formation of more predictable and stable antibody-parasite complexes. Antibody staining can be either direct or indirect. The direct technique utilizes an anti-parasite antibody conjugated with the fluorochrome (e.g., Musial et al., 1987). Indirect staining is a multistep process where the anti-parasite antibody is unlabeled and visualization occurs via a second, fluorescently labeled antibody that interacts with the first antibody bound to the parasite. In general, more background fluorescence can occur using direct techniques due to low level non-specific antibody binding. Nonetheless, immunofluorescence methods for the detection of Cryptosporidium and Giardia are commercially available and have been positively evaluated for sensitivity and specificity (Garcia and Shimizu, 1997; Graczyk et al., 1996; Lindquist et al., 2001). In one case, polyspecific antibodies cross-reactive with both Cryptosporidium and Giardia were developed with the idea that larger sample numbers could be quickly evaluated followed by more specific identification of positive specimens via PCR or species-specific monoclonal antibodies (El-Shewy and El-Hamshary, 1999). An indirect method developed by Ongerth and Stibbs (1987) used rabbit polyclonal antibodies to specifically bind Cryptosporidium previously purified from river water and still bound to the polycarbonate filters. The filters were secondarily treated with FITC-conjugated goat anti-rabbit IgG for detection via microscopy. More recent tests perform these analyses in solution rather than attached to a solid support to eliminate the steric hindrance that solid supports can impart on antigen-antibody binding and epitope conformation. Numerous similar reagents and protocol have been developed for immunofluorescent detection of Cryptosporidium and Giardia. Inclusion of the fluorogen $4^{\prime}, 6^{\prime}$-diamidino-2-phenylindole (DAPI) can facilitate positive identification of Cryptosporidia oocysts by staining sporozoite nuclei (Smith et al., 2002) and has become an integral part of USEPA Method 1623. In this work, the investigators concluded that combining DAPI-enhanced nuclei fluorescence (at least one nuclei) with oocyst wall fluorescence via fluorescein-labeled antibodies, increased the percentage of identifiable oocysts to $56 \%$ in the environmental samples tested, though this percentage dropped to $32 \%$ when the requirement for the number of identifiable nuclei was increased from 1 to 4 . Given the dissemination of these parasites and other disease agents even in conditioned swimming water (Fournier et al., 2002), and the potential impact of microsporidia infections in non-immunocompetent individuals, immunofluorescencebased detection methods for this group of parasites is also on the rise (Alfa Cisse et al., 2002; Beckers et al., 1996).

Even when using high quality reagents or commercially developed kits, staining techniques and protocols must be standardized and validated, and appropriate positive and negative controls included to ensure reliable and reproducible detection. This is of particular importance in dealing with background fluorescence even when using indirect 
detection methods. Rodgers et al. (1995) demonstrated that when examining water samples, the ASTM indirect immunofluorescence assay for staining Giardia/Cryptosporidium cross-reacted with many common algae. Although this problem was circumvented with the addition of goat serum, the inclusion of blocking agents is not always successful in eliminating background. Thus standardization and validation of assays including commercially available kits, are required for each sample type.

\subsection{Flow cytometry}

In a flow cytometer, particles in suspension flow in single file through a laser beam. As the particle intersects the beam, the laser light is scattered and fluorescence may be emitted by the particle or by a fluorescent stain specific for the organism of interest. The flow cytometer then collects the information and produces a profile of the particle suspension whereby all particles possessing similar characteristics cluster together. In epidemiological studies, a combination of IMS and flow cytometry was shown by Power et al. (2003) to better predict Cryptosporidium prevalence and oocyst shedding intensity.

Modern flow cytometers also have the ability to sort a suspension of particles based on one or more characteristics selected by the operator. Flow cytometry analysis has been used with water samples to detect Cryptosporidium oocysts (Vesey et al., 1991) and Giardia cysts (Dixon et al., 1997). While it was found that the sensitivity of the instruments was insufficient to distinguish oocysts from some background debris (Vesey et al., 1991), fluorescently labeled Giardia cysts were readily detected with only occasional poor resolution (Dixon et al., 1997). However, the fewer problems associated with contaminating debris may have greater foundation in the larger size of the Giardia cysts than in the methodology itself. The incorporation of cell sorting with flow cytometry addressed this issue (Vesey et al., 1993b) and was shown applicable to Giardia cysts as well (Medema et al., 1998 Vesey et al., 1994). In general, water samples are concentrated and stained in suspension with FITC-labeled antibodies then passed through the fluorescence activated cell sorter (FACS). Particles with the fluorescence and light-scattering characteristics of oocysts and cysts are sorted from the sample stream and collected on a microscope slide or membrane filter, which can then be examined by fluorescence microscopy to confirm the presence of parasites. While quite useful as a screening technique, FCM is not sufficiently specific or sensitive to obtain accurate counts of the organisms present. Other organisms and particles of similar size may cross react with the monoclonal antibody and have similar fluorescence characteristics. Additionally, some water samples that contain autofluorescent algae similar in size to oocysts and cysts to lead to incorrect conclusions if the FACS is used directly to produce parasite counts. However, confirmation by microscopy can be performed more easily and reliably than direct microscopy of unsorted samples. Research from France, the Netherlands, and the United States have confirmed the benefits of FACS for examining water samples for the presence of oocysts. (Compagnon, 1997; Danielson et al., 1995; Medema et al., 1998). FACS is currently being used for water analysis in the United Kingdom and is becoming more widely accepted in other parts of Europe, Australia, and South Africa. Recent advances in machine design and capabilities may result in increased utility of flow cytometry for 
sample analysis and in so doing provide more accurate oocyst counts; however, current technology appears limited to well purified samples from non-environmental sources.

\subsection{Molecular techniques}

The use of repetitive DNA hybridization probes and restriction fragment length polymorphism (RFLP) of total genomic DNA were among the first molecular techniques used for parasite identification where RFLPs were visualized either by direct examination of ethidium bromide stained gels or through hybridization of labeled probes. Applications of these and other macromolecular identification methods have declined in recent years and are being replaced in the more developed countries by the safety, enhanced sensitivity, and specificity of DNA sequencing, PCR, and their trailing technologies, including but not limited to real-time PCR. One such technology which utilized randomly derived PCR primers (RAPD) to generate low stringency agarose gel-banding profiles characteristic of a given parasite group, was found to be laden with technological barriers resulting in false profiles, low reproducibility, and could only be evaluated using highly purified samples. Consequently, its utilization for detecting environmentally derived samples met with little success.

In general, detection of parasites using molecular methods has not been widely accepted for commercial development even though they can provide substantial benefits over the more commonplace microscopic techniques. The largest obstacles to their development are excessive experimental manipulation, the perceived lack of necessity for higher sensitivities and specificities, the need for adequate controls, and circumventing problems relating to environmental inhibitors present in samples. Poorly characterized but potent inhibitors that either bind to PCR substrates or to the thermostable enzyme itself, in many cases are negatively charged and therefore co-purify with the parasite DNA using many conventional purification techniques. The presence of even trace amounts of inhibitors requires considerable dilution, extensive DNA purification or the addition of scavengers such as bovine serum albumin (Giambernardi et al., 1998; Kreader, 1996) to enhance the PCR. These, in turn, reduce the sensitivity of the assay and add additional steps to the overall process.

Numerous techniques have been developed to purify genomic DNA. Most involve binding and elution of the DNA from a support resin. These techniques are very efficient in the isolation of PCR fragments or in the preparation of plasmid DNA; however, quantitatively retrieving resin bound genomic DNA can be a formidable task that ultimately effects sensitivity. Nonetheless, many groups have successfully integrated one or more of the purification methods described above with PCR to detect parasites in environmental samples. The most common method is to combine immunocapture of parasites with PCR (IC-PCR) (Deng et al., 1997; Rimhanen-Finne et al., 2001, 2002) or with reverse transcription followed by PCR (IC-RT PCR). These detection schemes use either conventional gel analysis or real-time PCR for final assignment.

A plethora of PCR based tests exist to identify parasites, each varying by the choice of purification schemes, primer sets, target molecules or amplification protocols. Techniques capable of differentiating between human and animal pathogens, and thereby defining the risks of human infection from water supplies or local environments provide the biggest 
advancements to current tests. Thus, it is no longer sufficient to generically identify Cryptosporidium or Giardia in water supplies, but has become more important to define the genotypes or species as human or animal pathogens. This level of identification is not available using currently accepted methodology and provides the impetus for implementing newer more specific detection methods. For example, Amar et al. (2002) were able to differentiate assemblages $\mathrm{A}$ and $\mathrm{B}$ from $G$. duodenalis and further differentiate groups I and II within assemblage A by targeting the triose phosphate isomerase gene using a nested PCR-RFLP approach. Later, Amar et al. (2003) expanded on this work by combining a conventional, multiplex PCR format with two subsequent real-time PCR assays to delineate these same assemblages. Both test formats claimed specificity, sensitivity and reproducibility; yet, such assays are not likely to be accepted as commercial ventures given their multi-layered protocols. On the other hand, Johnson et al. (2003) targeted the naturally enriched small subunit ribosomal DNA and used sequence-specific fluorescent probes to distinguish groups I and II from $G$. duodenalis via real-time PCR. Similar examples of the usefulness of PCR can be found in assays to differentiate the bovine (C. parvum and the recently named human $C$. hominis) species of Cryptosporidium.

Combining assays for different parasite groups permits initial scanning of large sample numbers and leaves more discriminatory analyses to be performed only on the positive samples thereby reducing the work load. Multiplex PCR is one method, which is amenable to such an approach. Rochelle et al. (1997) were able to simultaneously differentiate $C$. parvum and $G$. lamblia in water samples by targeting the $18 \mathrm{~S}$ rRNA gene of Cryptosporidium and the heat shock protein gene of Giardia, followed by gel electrophoresis. Detection sensitivities ranged from 1 to 10 oocysts or cysts for purified preparations and 5-50 oocysts or cysts for seeded environmental waters, though maximum sensitivities were only obtained following two rounds of PCR and hybridization with chemiluminescent oligonucleotide probes. Lindergard et al. (2003) further delineated Cryptosporidium species and genotypes using a nested, multiplex PCR of the heat shock protein 70 . Detection limits varied between 10 and 100 oocysts $/ 1 \mathrm{ml}$ of fecal material, though to achieve this level of sensitivity, multiple PCRs were required as in the method by Rochelle et al. (1997). Work focusing on multiple alleles associated with the dihydrofolate reductase gene of Cryptosporidium provided equal or greater sensitivity than PCR-RFLP or nested PCR assays for detecting genotypes 1 and 2 (now delineated as $C$. parvum and C. hominis, respectively) (Gile et al., 2002). The one-step reaction was simpler and less expensive to perform than the RFLP methods and final assignment was made by agarose gel electrophoresis. Hester et al. (2002) took a unique approach to detecting three Encephalitozoon species using a fluorogenic assay. Species-specific primers were devised that amplify Encephalitozoon hellem, E. cuniculi or E. intestinalis and a single, genusspecific, dual fluorescent-labeled probe complementary to a conserved region within the Encephalitozoon 16S rDNA that was used for final detection. In this way, the assay could be tailored for general detection of Encephalitozoon parasites within a given sample by including all three primer pairs, or used for species level detection by including only single primer pair.

As with any assay, and in particular those, which are PCR based, proper controls are a requisite for reliable and reproducible testing. Kaucner and Stinear (1998) developed a novel type of internal positive control for Giardia and Cryptosporidium to account for 
problems associated with inhibitors that co-purify with the parasites, with isolating RNA, and with the subsequent enzymology associated with cDNA synthesis and PCR. In rather unique circumstances, positive internal controls can be built into a PCR assay. Zarlenga et al. (1999) used variability within expansion segment V of the large subunit ribosomal DNA to design multiplex primers to differentiate all species and genotypes of Trichinella. In so doing, one set of primers was designed to work on all genotypes. In this case, a negative reaction is then indicative of a problem with the assay rather than a negative sample.

\section{Methodologies on the horizon}

Methods are currently being devised to circumvent the two-step gel assays for detecting the plethora of parasitic agents in environmental samples. These involve developing more sensitive real-time PCR assays in 96 and 384 well format, and using machines capable of multiplexing fluorescent signals. Real-time assays have been developed for microsporidia species (Menotti et al., 2003; Wolk et al., 2002), Cryptosporidium (Fontaine and Guillot, 2002; Higgins et al., 2001; Limor et al., 2002), Giardia (Amar et al., 2003), Toxoplasma (Jauregui et al., 2001; Lin et al., 2000), and a number of nematode and cestode species whose eggs can survive environmental stress for long periods of time. Also, more sophisticated software is now available to assist in generating multiplex compatible primers and sequence-specific fluorescent probes. Combining multiplex PCR with microarray scanning is currently being explored for high throughput screening and detection with special emphasis on gene expression profiling, testing for human disease characteristics, and for single-nucleotide polymorphism genotyping. However, one can envision adapting this technology to amplified genomic DNA rather than cDNA for simultaneously detecting multiple parasitic agents. Such a technology will also permit quantitation of disease agents in a particular sample.

Solving problems with environmental sampling will require innovative approaches. Automation of magnetic bead technology is one area that can move the union of PCR technology and environmental sampling closer to realization. Magtration ${ }^{\mathrm{TM}}$ produced by PSS Bio Instruments involves full instrumentation in 96 well format to purify samples using magnetic bead technology. Currently it is optimized for DNA isolation; however, it may be possible to adapt the technology to immunocapture. This methodology includes a single instrument that poses low risk of sample cross-over and user infection, and is specifically designed for high throughput diagnostic purposes in 96 well format. Currently it is capable of processing 5000 samples daily.

A third technology on the horizon that does not involve PCR is the use of gold nanoparticles for detection. In this technology, gold particles $\left(0.001\right.$ particles $\left./ \mu^{2}\right)$ are modified with high density oligos specifically complementary to the sequence of interest. When attached to the particles, the oligos retain their recognition properties for the target sequence, are stable to salt and temperature changes, and exhibit low non-specific binding. Once DNA is extracted from the purified environmental sample, the oligo-impregnated, gold nanoparticles are added and bound up by the DNA. This mixture is then hybridized to an array containing capture probes for the specific oligos present on the gold particles. The array is finally enhanced with silver and a scatter image is taken. Presence is indicated by a 
shift in the color of the gold particles. Sensitivities are several fold greater than current fluorescent based methods for detection. Though originally designed for direct detection of target DNA, implementing a truncated PCR step could substantially enhance the prospects of this technology for low level pathogen detection.

It is possible that matrix-assisted laser desorption time of flight (MALDI-TOF) mass spectrometry may one day find application in testing environmental samples. In 2001, Fenselau and Demirev (2001) reviewed the state of the technology for identifying and desorbing protein biomarkers that are unique and representative of specific intact viruses, bacteria, fungus, and spores. Later, Demirev et al. (2002) showed that by focusing on the spectrum of ferriprotoporphyrin IX (heme), which is structure specific and which is sequestered by Plasmodium during their growth in human red cells, they could detect parasitemia at the level of 10 parasites/mL of blood. Most recently, Grant et al. (2003) were among the first to detect trace-levels of drug residues in complex environmental samples using immunoextraction techniques followed by MALDI-TOF mass spectrometry. Though detecting drugs is quite different from deciphering biomolecule profiles of parasites, it nonetheless supports the importance of working with clean preparations and demonstrates the potential to apply this technology to test environmental samples. As with the work of Demirev et al. (2002), Grant et al. (2003) show that identifying biomarkers unique to specific genera of parasites will be the limiting factor in adapting this technology to organisms substantially more complicated than bacteria, fungi and viruses.

Detecting parasites in environmental samples is a field that is still evolving, though the process is substantially based in technological advances driven by human research. Detection methods of the future will likely require newer, more sophisticated assays; however, the potential to adapt, refine and adjoin older methods with those currently in use or development must not be overlooked in achieving these goals. As our understanding of relationships among parasite groups increases and the phylogeny of environmentally derived pathogens is clarified, accurate detection becomes more imperative and more difficult as well. Training laboratory personnel in state-of the art techniques, optimizing sample preservation and processing, controlling reagent and instrumentation costs, and dealing with data interpretation and troubleshooting are among the many obstacles that will be encountered in the application of new molecular-based methods. Nonetheless, societal demands for safer living conditions, threats imposed by bioterrorism, and global distribution of the most harmful human parasites are compelling reasons to continue the quest for more sensitive and accurate detection methods.

\section{Acknowledgment}

We thank Dr. R. Fayer for critical review of the manuscript.

\section{References}

Alfa Cisse, O., Ouattara, A., Thellier, M., Accoceberry, I., Biligui, S., Minta, D., Doumbo, O., Desportes-Livage, I., Thera, M.A., Danis, M., Datry, A., 2002. Evaluation of an immunofluorescent-antibody test using 
monoclonal antibodies directed against Enterocytozoon bieneusi and Encephalitozoon intestinalis for diagnosis of intestinal microsporidiosis in Bamako (Mali). J. Clin. Microbiol. 40, 1715-1718.

Amar, C.F., Dear, P.H., McLauchlin, J., 2003. Detection and genotyping by real-time PCR/RFLP analyses of Giardia duodenalis from human faeces. J. Med. Microbiol. 52, 681-683.

Amar, C.F., Dear, P.H., Pedraza-Diaz, S., Looker, N., Linnane, E., McLauchlin, J., 2002. Sensitive PCR-restriction fragment length polymorphism assay for detection and genotyping of Giardia duodenalis in human feces. J. Clin. Microbiol. 40, 446-452.

Anderson, B.C., 1986. Effect of drying on the infectivity of cryptosporidia-laden calf feces for 3- to 7-day old mice. Am. J. Vet. Res. 47, 2272-2273.

Bautista, E., Pezzani, B.C., Cordoba, A., De Luca, M.M., Basualdo, J.A., 1999. Relationship between discontinuous sucrose gradient and viability of Cryptosporidium sp. oocysts. Rev. Argent. Microbiol. 31, 188192 (in Portuguese).

Beckers, P.J., Derks, G.J., van Gool, T., Rietveld, F.J., Sauerwein, R.W., 1996. Encephalocytozoon intestinalisspecific monoclonal antibodies for laboratory diagnosis of microsporidiosis. J. Clin. Microbiol. 34, $282-285$.

Becnel, J.J., Andreadis, T.G., 1999. Microsporidia in insects. In: Wittner, M., Weiss, L.M. (Eds.), The Microsporidia and Microsporidiosis. ASM Press, Washington, DC, pp. 447-501.

Bialek, R., Binder, N., Dietz, K., Knobloch, J., Zelck, U.E., 2002. Comparison of autofluorescence and iodine staining for detection of Isospora belli in feces. Am. J. Trop. Med. Hyg. 67, 304-305.

Borchardt, M.A., Spencer, S.K., 2002. Concentration of Cryptosporidium, microsporidia and other water-borne pathogens by continuous separation channel centrifugation. J. Appl. Microbiol. 92, 649-656.

Boveri, T., 1887. Uber differenzierung of zelkerne wahrend der furchung des eies of Ascaris megalocephala. Anat. Ang. 2, 688-693.

Brandonisio, O., Portincasa, F., Torchetti, G., Lacarpia, N., Rizzi, A., Fumarola, L., Donadio, F., Carnimeo, D., 2000. Giardia and Cryptosporidium in water: evaluation of two concentration methods and occurrence in wastewater. Parasitologia 42, 205-209.

Bukhari, Z., McCuin, R.M., Fricker, C.R., Clancy, J.L., 1998. Immunomagnetic separation of Cryptosporidium parvum from source water samples of various turbidities. Appl. Environ. Microbiol. 64, 4495-4499.

Bukhari, Z., Smith, H.V., 1995. Effect of three concentration techniques on viability of Cryptosporidium parvum oocysts recovered from bovine feces. J. Clin. Microbiol. 33, 2592-2595.

Campbell, A., Smith, H., 1997. Immunomagnetic separation of Cryptosporidium oocysts from water samples: round robin comparison of techniques. Wat. Sci. Technol. 35, 397-401.

Campbell, A.T., Gron, B., Johnsen, S.E., 1997. Immunomagnetic separation of Cryptosporidium oocysts from high turbidity water sample concentrates. In: Fricker, C.R., Clancy, J.L., Rochelle, P.A. (Eds.), Proceedings of the International Symposium on Waterborne Cryptosporidium. AWWA, Denver, CO, pp. 91-96.

Campbell, A.T., Robertson, L.J., Smith, H.V., Girdwood, R.W.A., 1994. Viability of Cryptosporidium parvum oocysts concentrated by calcium carbonate flocculation. J. Appl. Bacteriol. 76, 638-639.

Clancy, J.L., Gollnitz, W.D., Tabib, Z., 1994. Commercial labs: how accurate are they? J. Am. Water Works Assoc. 86, 89-97.

Clancy, J.L., Hargy, T.M., Schaub, S., 1997. Improved sampling methods for the recovery of Giardia and Cryptosporidium from source and treated water. In: Fricker, C.R., Clancy, J.L., Rochelle, P.A. (Eds.), Proceedings of the International Symposium on Waterborne Cryptosporidium. AWWA, Denver, CO, pp. 79-85.

Compagnon, B., 1997. Improved detection of Giardia cysts and Cryptosporidium oocysts in water by flow cytometry. In: Alvarez, M.B. (Ed.), Proceedings of the Water Quality Technology Conference, AWWA, Denver, CO.

Danielson, R.E., Cooper, R.C., Riggs, J.L., 1995. Giardia and Cryptosporidium analysis: a comparison of microscopic and flow cytometric techniques. In: Proceedings of the Water Quality Technology Conference. AWWA, Denver, CO, pp. 1673-1685.

Daugschies, A., Bialek, R., Joachim, A., Mundt, H.C., 2001. Autofluorescence microscopy for the detection of nematode eggs and protozoa, in particular Isospora suis, in swine faeces. Parasitol. Res. 87, 409-412.

Demirev, P.A., Feldman, A.B., Kongkasuriyachai, D., Scholl, P., Sullivan Jr., D., Kumar, N., 2002. Detection of malaria parasites in blood by laser desorption mass spectrometry. Anal. Chem. 74, 3262-3266.

Deng, M.Q., Cliver, D.O., Mariam, T.W., 1997. Immunomagnetic capture PCR to detect viable Cryptosporidium parvum oocysts from environmental samples. Appl. Environ. Microbiol. 63, 3134-3138. 
deRegnier, D.P., Cole, L., Schupp, D.G., Erlandsen, S.L., 1989. Viability of Giardia cysts suspended in lake, river, and tap water. Appl. Environ. Microbiol. 55, 1223-1229.

Dixon, B.R., Parenteau, M., Martineau, C., Fournier, J., 1997. A comparison of conventional microscopy, immunofluorescence microscopy and flow cytometry in the detection of Giardia lamblia cysts in beaver fecal samples. J. Immunol. Meth. 202, 27-33.

Dowd, S.E., Gerba, C.P., Enriquez, F.J., Pepper, I.L., 1998. PCR amplification and species determination of microsporidia in formalin-fixed feces after immunomagnetic separation. Appl. Environ. Microbiol. 64, 333336.

Drozd, C., Schwartzbrod, J., 1996. Hydrophobic and electrostatic cell surface properties of Cryptosporidium parvum. Appl. Environ. Microbiol. 62, 1227-1232.

El-Shewy, K.A., El-Hamshary, E.M., 1999. Immunofluorescent detection of both Giardia lamblia and Cryptosporidium parvum using anti-Cryptosporidium oocyst antibodies. J. Egypt Soc. Parasitol. 29, 777-786.

Entrala, E., Molina-Molina, J., Rosales-Lombardo, M., Sanchez-Moreno, M., Mascaro-Lazcano, C., 2000. Cryptosporidium parvum: oocysts purification using potassium bromide discontinuous gradient. Vet. Parasitol. 92, 223-226.

Fayer, R., Nerad, T., 1996. Effects of low temperatures on viability of Cryptosporidium parvum oocysts. Appl. Environ. Microbiol. 62, 1431-1433.

Fayer, R., Santin, M., Trout, J.M., 2003. First detection of microsporidia in dairy calves in North America. Parasitol. Res. 90, 383-386.

Fayer, R., Trout, J.M., Jenkins, M.C., 1998. Infectivity of Cryptosporidium parvum oocysts stored in water at environmental temperatures. J. Parasitol. 84, 1165-1169.

Feng, Y.Y., Ong, S.L., Hu, J.Y., Song, L.F., Tan, X.L., Ng, W.J., 2003. Effect of particles on the recovery of Cryptosporidium oocysts from source water samples of various turbidities. Appl. Environ. Microbiol. 69, 1898-1903.

Fenselau, C., Demirev, P.A., 2001. Characterization of intact microorganisms by MALDI mass spectrometry. Mass Spectrom. Rev. 20, 157-171.

Ferrari, B.C., Veal, D., 2003. Analysis-only detection of Giardia by combining immunomagnetic separation and two-color flow cytometry. Cytometry 51A, 79-86.

Fontaine, M., Guillot, E., 2002. Development of a TaqMan quantitative PCR assay specific for Cryptosporidium parvum. FEMS Microbiol. Lett. 214, 7-13.

Fournier, S., Dubrou, S., Liguory, O., Gaussin, F., Santillana-Hayat, M., Sarfati, C., Molina, J.M., Derouin, F., 2002. Detection of Microsporidia Cryptosporidia and Giardia in swimming pools: a one-year prospective study. FEMS Immunol. Med. Microbiol. 33, 209-213.

Fricker, C.R., Jonas, A., Crabb, J., Turner, N., Smith, H.V., 1997. The concentration and separation of Cryptosporidium oocysts and Giardia cysts using vortex flow filtration and immunomagnetic separation. In: Fricker, C.R., Clancy, J.L., Rochelle, P.A. (Eds.), Proceedings of the International Symposium on Waterborne Cryptosporidium. AWWA, Denver, CO, pp. 1-8.

Garcia, L.S., Shimizu, R.Y., 1997. Evaluation of nine immunoassay kits (enzyme immunoassay and direct fluorescence) for detection of Giardia lamblia and Cryptosporidium parvum in human fecal specimens. J. Clin. Microbiol. 35, 1526-1529.

Giambernardi, T.A., Ulrich, R., Klebe, R.J., 1998. Bovine serum albumin reverses inhibition of RT-PCR by melanin. Biotechniques 25, 564-566.

Gile, M., Warhurst, D.C., Webster, K.A., West, D.M., Marshall, J.A., 2002. A multiplex allele specific polymerase chain reaction (MAS-PCR) on the dihydrofolate reductase gene for the detection of Cryptosporidium parvum genotypes 1 and 2. Parasitology 125 (Pt 1), 35-44.

Graczyk, T.K., Cranfield, M.R., Fayer, R., 1996. Evaluation of commercial enzyme immunoassay (EIA) and immunofluorescent antibody (FA) test kits for detection of Cryptosporidium oocysts of species other than Cryptosporidium parvum. Am. J. Trop. Med. Hyg. 54, 274-279.

Graczyk, T.K., Fayer, R., Cranfield, M.R., Mhangami-Ruwende, B., Knight, R., Trout, J.M., Bixler, H., 1999. Filth flies are transport hosts of Cryptosporidium parvum. Emerg. Infect. Dis. 5, 726-727.

Graczyk, T.K., Fayer, R., Knight, R., Mhangami-Ruwende, B., Trout, J.M., DaSilva, A.J., Pieniazek, N.J., 2000. Mechanical transport and transmission of Cryptosporidium parvum oocysts by wild filth flies. Am. J. Trop. Med. Hyg. 63, 178-183. 
Grant, G.A., Frison, S.L., Sporns, P.J., 2003. A sensitive method for detection of sulfamethazine and N(4)acetylsulfamethazine residues in environmental samples using solid phase immunoextraction coupled with MALDI-TOF MS. Agric. Food Chem. 51, 5367-5375.

Hester, J.D., Varma, M., Bobst, A.M., Ware, M.W., Lindquist, H.D., Schaefer, F.W., 2002. Species-specific detection of three human-pathogenic microsporidial species from the genus Encephalitozoon via fluorogenic 5 ' nuclease PCR assays. Mol. Cell. Probes 16, 435-444.

Higgins, J.A., Fayer, R., Trout, J.M., Xiao, L., Lal, A.A., Kerby, S., Jenkins, M.C., 2001. Real-time PCR for the detection of Cryptosporidium parvum. J. Microbiol. Meth. 47, 323-337.

Higgins, J.A., Trout, J.M., Fayer, R., Shelton, D., Jenkins, M.C., 2003. Recovery and detection of Cryptosporidium parvum oocysts from water samples using continuous flow centrifugation. Water Res. 37, 3551-3560.

Hsu, B.M., 2003. Evaluation of analyzing methods for Giardia and Cryptosporidium in a Taiwan water treatment plant. J. Parasitol. 89, 369-371.

Hsu, B.M., Huang, C., Hsu, Y.F., Jiang, G.Y., Hsu, C.L., 2001a. Evaluation of two concentration methods for detecting Giardia and Cryptosporidium in water. Water Res. 35, 419-424.

Hsu, B.M., Huang, C., Pan, J.R., 2001b. Filtration behaviors of Giardia and Cryptosporidium-ionic strength and pH effects. Water Res. 35, 3777-3782.

Jauregui, L.H., Higgins, J., Zarlenga, D., Dubey, J.P., Lunney, J.K., 2001. Development of a real-time PCR assay for detection of Toxoplasma gondii in pig and mouse tissues. J. Clin. Microbiol. 39, 2065-2071.

Johnson, M.L., Berryman, D.I., Reynoldson, J.A., Thompson, R.C., 2003. A fluorescent based PCR assay for the detection and quantitation of Giardia duodenalis genotypes in mixed populations. Infect. Genet. E 3, 97-102.

Kaucner, C., Stinear, T., 1998. Sensitive and rapid detection of viable Giardia cysts and Cryptosporidium parvum oocysts in large-volume water samples with wound fiberglass cartridge filters and reverse transcription-PCR. Appl. Environ. Microbiol. 64, 1743-1749.

Kreader, C.A., 1996. Relief of amplification inhibition in PCR with bovine serum albumin or T4 gene 32 protein. Appl. Environ. Microbiol. 62, 1102-1106.

Kuczynska, E., Shelton, D., 1999. Method for detection and enumeration of Cryptosporidium parvum oocysts in feces, manures, and soils. Appl. Environ. Microbiol. 65, 2820-2826.

Kuhn, R.C., Oshima, K.H., 2002. Hollow-fiber ultrafiltration of Cryptosporidium parvum oocysts from a wide variety of $10 \mathrm{~L}$ surface water samples. Can. J. Microbiol. 48, 542-549.

Limor, J.R., Lal, A.A., Xiao, L., 2002. Detection and differentiation of Cryptosporidium parasites that are pathogenic for humans by real-time PCR. J. Clin. Microbiol. 40, 2335-2338.

Lin, M.H., Chen, T.C., Kuo, T.T., Tseng, C.C., Tseng, C.P., 2000. Real-time PCR for quantitative detection of Toxoplasma gondii. J. Clin. Microbiol. 38, 4121-4125.

Lindergard, G., Nydam, D.V., Wade, S.E., Schaaf, S.L., Mohammed, H.O., 2003. A novel multiplex polymerase chain reaction approach for detection of four human infective Cryptosporidium isolates: Cryptosporidium parvum, types $\mathrm{H}$ and C, Cryptosporidium canis, and Cryptosporidium felis in fecal and soil samples. J. Vet. Diagn. Invest. 15, 262-267.

Lindquist, H.D., Ware, M., Stetler, R.E., Wymer, L., Schaefer III, F.W., 2001. A comparison of four fluorescent antibody-based methods for purifying, detecting, and confirming Cryptosporidium parvum in surface waters. J. Parasitol. 87, 1124-1131.

Malicki, J., Montusiewicz, A., Bieganowski, A., 2001. Improvement of counting helminth eggs with internal standard. Water Res. 35, 2333-2335.

Medema, G.J., Shets, F.M., Ketelaars, H., Boschman, G., 1998. Improved detection and vital staining of Cryptosporidium and Giardia with flow cytometry. Wat. Sci. Tech. 38, 61-65.

Menotti, J., Cassinat, B., Porcher, R., Sarfati, C., Derouin, F., Molina, J.M., 2003. Development of a real-time polymerase-chain-reaction assay for quantitative detection of Enterocytozoon bieneusi DNA in stool specimens from immunocompromised patients with intestinal microsporidiosis. J. Infect. Dis. 187, 1469-1474.

Mes, T.H., Ploeger, H.W., Terlou, M., Kooyman, F.N., Van der Ploeg, M.P., Eysker, M., 2001. A novel method for the isolation of gastro-intestinal nematode eggs that allows automated analysis of digital images of egg preparations and high throughput screening. Parasitology 123, 309-314.

Musial, C.E., Arrowood, M.J., Sterling, C.R., Gerba, C.P., 1987. Detection of Cryptosporidium in water by using polypropylene cartridge filters. Appl. Environ. Microbiol. 53, 687-692. 
Nieminski, E.C., Schaefer III, F.W., Ongerth, J.E., 1995. Comparison of two methods for detection of Giardia cysts and Cryptosporidium oocysts in water. Appl. Environ. Microbiol. 61, 1714-1719.

Ongerth, J.E., Stibbs, H.H., 1987. Identification of Cryptosporidium oocysts in river water. Appl. Environ. Microbiol. 53, 672-676.

Power, M.L., Shanker, S.R., Sangster, N.C., Veal, D.A., 2003. Evaluation of a combined immunomagnetic separation/flow cytometry technique for epidemiological investigations of Cryptosporidium in domestic and Australian native animals. Vet. Parasitol. 112, 21-31.

Rimhanen-Finne, R., Horman, A., Ronkainen, P., Hanninen, M.L., 2002. An IC-PCR method for detection of Cryptosporidium and Giardia in natural surface waters in Finland. J. Microbiol. Meth. 50, 299-303.

Rimhanen-Finne, R., Ronkainen, P., Hanninen, M.L., 2001. Simultaneous detection of Cryptosporidium parvum and Giardia in sewage sludge by IC-PCR. J. Appl. Microbiol. 91, 1030-1035.

Robertson, L.J., Campbell, A.T., Smith, H.V., 1992. Survival of Cryptosporidium parvum oocysts under various environmental pressures. Appl. Environ. Microbiol. 58, 3494-3500.

Robertson, L.J., Gjerde, B., 2000. Isolation and enumeration of Giardia cysts, Cryptosporidium oocysts, and Ascaris eggs from fruits and vegetables. J. Food Protect. 63, 775-778.

Robertson, L.J., Gjerde, B., Campbell, A.T., 2000. Isolation of Cyclospora oocysts from fruits and vegetables using lectin-coated paramagnetic beads. J. Food Protect. 63, 1410-1414.

Rochelle, P.A., De Leon, R., Johnson, A., Stewart, M.H., Wolfe, R.L., 1999. Evaluation of immunomagnetic separation for recovery of infectious Cryptosporidium parvum oocysts from environmental samples. Appl. Environ. Microbiol. 65, 841-845.

Rochelle, P.A., De Leon, R., Stewart, M.H., Wolfe, R.L., 1997. Comparison of primers and optimization of PCR conditions for detection of Cryptosporidium parvum and Giardia lamblia in water. Appl. Environ. Microbiol. 63, 106-114.

Rodgers, M.K., Flanigan, D.J., Jukubowski, W., 1995. Identification of algae which interfere with the detection of Giardia cysts and Cryptosporidium oocysts and a method for alleviating this interference. Appl. Environ. Microbiol. 61, 3759-3763.

Shepherd, K.M., Wyn-Jones, A.P., 1996. An evaluation of methods for the simultaneous detection of Cryptosporidium oocysts and Giardia cysts from water. Appl. Environ. Microbiol. 62, 1317-1322.

Simmons III, O.D., Sobsey, M.D., Heaney, C.D., Schaefer III, F.W., Francy, D.S., 2001. Concentration and detection of Cryptosporidium oocysts in surface water samples by method 1622 using ultrafiltration and capsule filtration. Appl. Environ. Microbiol. 67, 1123-1127.

Smith, H.V., Campbell, B.M., Paton, C.A., Nichols, R.A., 2002. Significance of enhanced morphological detection of Cryptosporidium sp. oocysts in water concentrates determined by using 4',6'-diamidino-2-phenylindole and immunofluorescence microscopy. Appl. Environ. Microbiol. 68, 5198-5201.

Sorel, N., Guillot, E., Thellier, M., Accoceberry, I., Datry, A., Mesnard-Rouiller, L., Miegeville, M., 2003. Development of an immunomagnetic separation-polymerase chain reaction (IMS-PCR) assay specific for Enterocytozoon bieneusi in water samples. J. Appl. Microbiol. 94, 273-279.

Sprague, V., Becnel, J.J., 1999. Appendix: checklist of available generic names for microsporidia with type species and type hosts. In: Wittner, M., Weiss, L.M. (Eds.), The Microsporidia and Microsporidiosis. ASM Press, Washington, DC, pp. 517-530.

Thompson, R.C.A., Hopkins, R.M., Homan, W.L., 2000. Nomenclature and genetic groupings of Giardia infecting mammals. Parasitol. Today 16, 210-213.

Tsushima, Y., Karanis, P., Kamada, T., Makala, L., Xuan, X., Tohya, Y., Akashi, H., Nagasawa, H., 2003. Seasonal change in the number of Cryptosporidium parvum oocysts in water samples from the rivers in Hokkaido, Japan, detected by the ferric sulfate flocculation method. J. Vet. Med. Sci. 65, 121-123.

U.S. Environmental Protection Agency, 1998. USEPA method 1622: Cryptosporidium in water by filtration/IMS/ IFA. EPA 821-R98010. Office of Water, U.S. Environmental Protection Agency, Washington, DC.

U.S. Environmental Protection Agency, 1999. Method 1623: Cryptosporidium and Giardia in water by filtration/ IMS/FA. EPA 821-R99006. Office of Water, U.S. Environmental Protection Agency, Washington, DC.

Vesey, G., Slade, J.S., Fricker, C.R., 1991. Taking the eye strain out of environmental Cryptosporidium analysis. Lett. Appl. Microbiol. 13, 62-65.

Vesey, G., Slade, J.S., Byrne, M., Shepherd, K., Fricker, C.R., 1993a. A new method for the concentration of Cryptosporidium oocysts from water. J. Appl. Bacteriol. 75, 82-86. 
Vesey, G., Slade, J.S., Byrne, M., Shepherd, K., Fricker, C.R., 1993b. Routine monitoring of Cryptosporidium oocysts in water using flow cytometry. J. Appl. Bacteriol. 75, 87-90.

Vesey, G., Hutton, P., Champion, A., Ashbolt, N., Williams, K.L., Warton, A., Veal, D., 1994. Application of flow cytometric methods for the routine detection of Cryptosporidium and Giardia in water. Cytometry 16, 1-6.

Wang, L.C., 1998. Improvement in the identification of intestinal parasites by a concentrated merthiolate-iodineformaldehyde technique. J. Parasitol. 84, 457-458.

Whitmore, T.N., Carrington, E.G., 1993. Comparison of methods for recovery of Cryptosporidium from water. Wat. Sci. Tech. 27, 69-76.

Wolk, D.M., Schneider, S.K., Wengenack, N.L., Sloan, L.M., Rosenblatt, J.E., 2002. Real-time PCR method for detection of Encephalitozoon intestinalis from stool specimens. J. Clin. Microbiol. 40, 3922-3928.

Xiao, L., Morgan, U.M., Fayer, R., Thompson, R.C., Lal, A.A., 2000. Cryptosporidium systematics and implications for public health. Parasitol. Today 16, 287-292.

Yakub, G.P., Stadterman-Knauer, K.L., 2000. Evaluation of immunomagnetic separation for recovery of Cryptosporidium parvum and Giardia duodenalis from high-iron matrices. Appl. Environ. Microbiol. 66, 3628-3631.

Zajac, A.M., Johnson, J., King, S.E., 2002. Evaluation of the importance of centrifugation as a component of zinc sulfate fecal flotation examinations. J. Am. Anim. Hosp. Assoc. 38, 221-224.

Zarlenga, D.S., Chute, M.B., Martin, A., Kapel, C.M., 1999. A multiplex PCR for unequivocal differentiation of all encapsulated and non-encapsulated genotypes of Trichinella. Int. J. Parasitol. 29, 1859-1867.

Zuckerman, U., Armon, R., Tzipori, S., Gold, D., 1999. Evaluation of a portable differential continuous flow centrifuge for concentration of Cryptosporidium oocysts and Giardia cysts from water. J. Appl. Microbiol. 86, 955-961. 